\title{
Multi Modal Fault Detection System of Virtual Instrument Test System Based on RBF Neural Network
}

\author{
Dian Zhang \\ Department of Foreign Languages, Hunan University of Humanities, Science \& Technology, Loudi \\ 417000, China \\ dianzhang_2013@yeah.net
}

Keywords: Neural network; Cognition; Virtual language; Simulation; Interactive; Image.

\begin{abstract}
This paper analyzes the interactive effects of virtual language multimodal through the use of neural network algorithm to understand the language from a multidimensional perspective, which mainly uses the advanced technology of digital language lab to improve its language proficiency. Through the analysis of virtual language simulation, we found that the multimodal virtual language can fully mobilize the visual, auditory, tactile and other sensory stimuli, and with the help of digital voice room vast resources, it can set sound, graphics, text and other information in one, which provides an interactive language environment, so as to meet the development requirements of language cognitive thinking.
\end{abstract}

\section{Introduction}

Multimodal study is currently an important and frontier academic field, which across multiple disciplines. Nowadays, the rapid development of corpus research and speech engineering as well as the continuous innovation of multimedia technology provide the powerful theory and technology support for the natural discourse research and multimodal study, which make their research possible [1-3]. People's daily communication not simply relies on a resource to achieve, but uses a variety of semiotic resources, so it has a multimodal. So this paper uses the neural network algorithm, the virtual language and the various language resource are combined to carry out the analysis of multi modal, the specific process is shown in Figure 1.

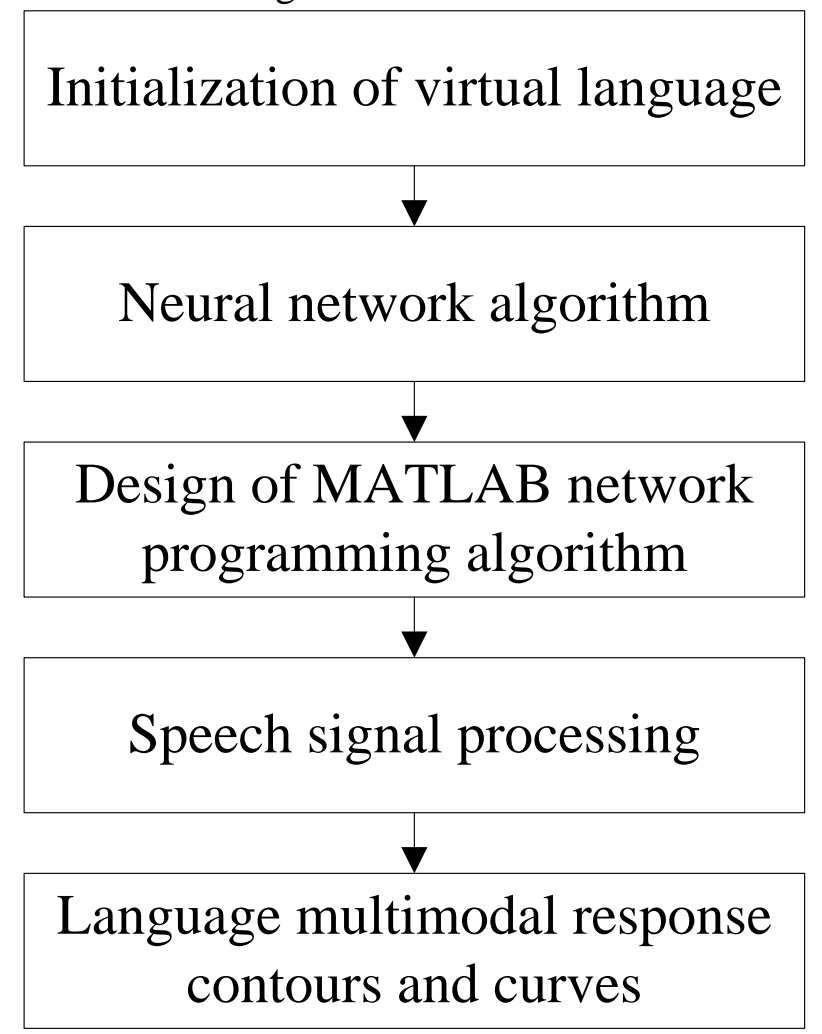

Fig.1: Schematic diagram of TEI model network language application 
Figure 1 shows that the calculation of language corpus is applied to virtual language multi modal system. From the chart, it is the basic process of neural network algorithm in the analysis of virtual language multi modal [4,5]. Based on the basic calculation language of virtual language database, we firstly establish the neural network training algorithm of language multi modal analysis errors, and then the use of software programming form carries on processing for the virtual language, finally we can obtain the language multi-modal response contours and curves.

\section{Multimodal Virtual Language Neural Network Algorithm}

For the multi modal analysis of language, it needs to combine the multi dimensionality of cognitive thinking to carry on pattern recognition for the single-layer perception, in which its expression is shown in formula (1).

$$
\sum_{i=1}^{m} w_{i} x_{i}+b=0
$$

So the multi modal model of virtual language can be expressed as:

$$
X(n)=\left[1, x_{1}(n), x_{2}(n), x_{3}(n), \cdots, x_{n}(n)\right] .
$$

And cognitive thinking dimensions can be represented as shown in formula (3).

$$
w(n)=\left[b(n), w_{1}(n), w_{2}(n), w_{3}(n), \cdots, w_{n}(n)\right] .
$$

Assumption that $y$ represents the expected output, then the expected output and actual error can be expressed as:

$$
e=d(n)-y(n) .
$$

For the multi-modal error of virtual language, it can be trained by using the neural network algorithm [6]. Neural network system is a biological output process, and its general is a multi input single output model as shown in Figure 2.

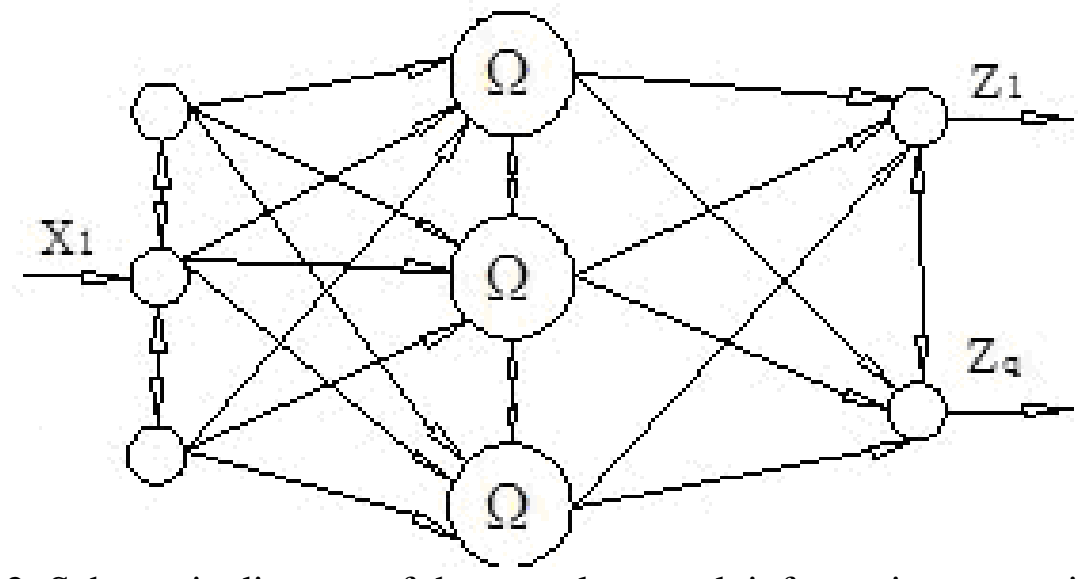

Fig.2: Schematic diagram of the neural network information transmission

Hypothesis that the input variables of neurons is $x_{i}(i=1,2, \cdots, m)$ and the output is $y_{j}$, then the relationship between input variables and output variables can be expressed as:

$$
\left\{\begin{array}{c}
s_{j}=\sum_{i=1}^{m} w_{i j} x_{i}-\theta_{j} \\
y_{j}=f\left(s_{j}\right)
\end{array} .\right.
$$

Where $j$ represents the threshold and $W_{i j}$ represents the connection weight factor from neuron $i$ to neuron $j$, they can use MATLAB software to program design for the neural network training algorithm, in which the use of main program algorithm are as follows $[7,8]$ :

nntwarn off

$\mathrm{XX}=\operatorname{premnmx}(\mathrm{XX})$;

$\mathrm{YY}=\operatorname{premnmx}(\mathrm{YY})$; 
$\mathrm{P}=\mathrm{XX}$;

$\mathrm{T}=\mathrm{YY}$;

$\mathrm{R}=\operatorname{size}(\mathrm{P}, 1)$;

$\mathrm{S} 2=\operatorname{size}(\mathrm{T}, 1)$;

$\mathrm{S} 1=25$;

$\mathrm{S}=\mathrm{R} * \mathrm{~S} 1+\mathrm{S} 1 * \mathrm{~S} 2+\mathrm{S} 1+\mathrm{S} 2 ; \%$

aa $=$ ones $(\mathrm{S}, 1) *[-1,1]$;

$\mathrm{popu}=50$;

\section{Virtual Language Multimedia Simulation}

In order to verify the effectiveness and reliability of multimodal language neural network analysis algorithm designed in the second part, this paper processes the virtual language signals using MATLAB software [9-11]. Through the MATLAB voice signal processing toolbox, we can obtain virtual language multi modal response curve.

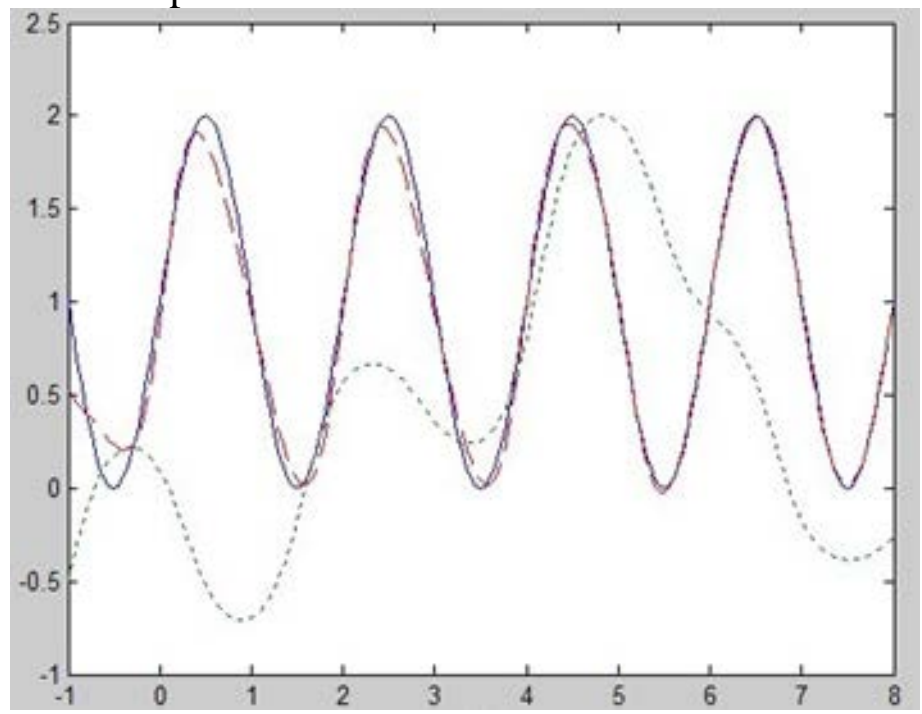

Fig.2: MATLAB toolbox voice signal

Figure 2 shows the three types of virtual language using MATLAB Simulink toolbox input, which mainly includes three kinds of language processing signal, they respectively correspond to the languages visual, auditory and tactile, and these signals can use the neural network iteration algorithm.

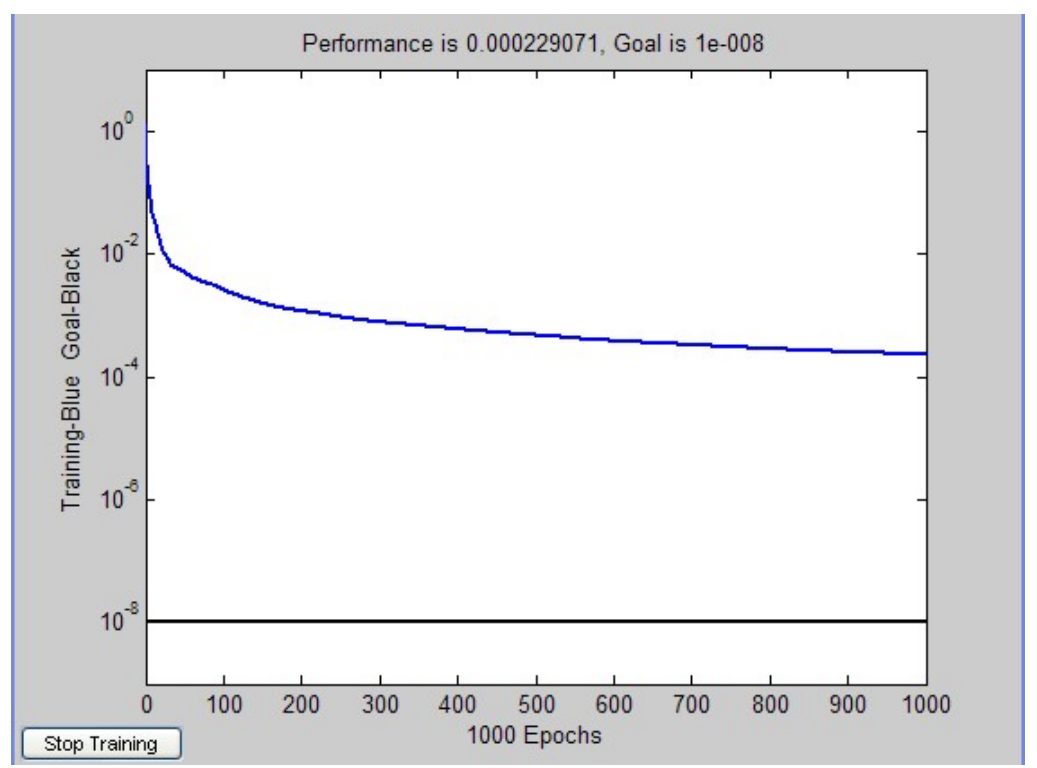

Fig.3: Neural network iterative convergence curve 
After the use of neural network algorithm carries out training for the virtual language signals, we can obtain the iterative convergence curve as shown in Figure 3. From the chart, it can be seen that when its iteration is 1000 steps, convergence error will be close to $10-4$, it is reached a stable convergence effect.

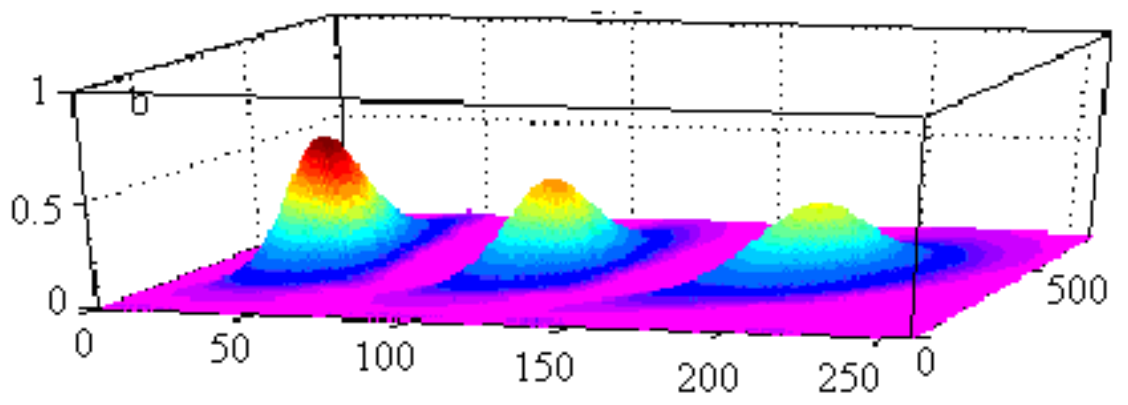

Fig.4: MATLAB multimodal response images

Figure 4 shows the virtual language signals analyzed by using MATLAB software and trained by neural network. After the virtual language signals reach stable convergence, we carry out the response analysis of visual, auditory and tactile signals, and then we can obtain the multimodal response images. The three peaks of the cloud respectively correspond to the language sounds, images and text mode.

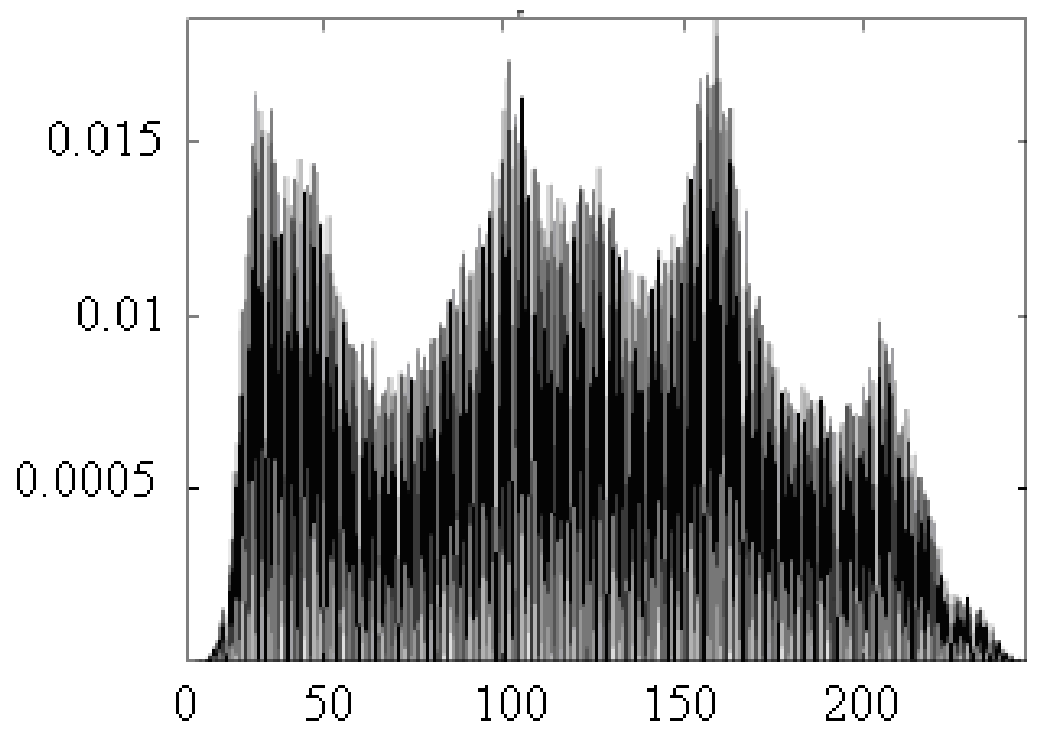

Fig.5: Multimodal response curve

Figure 5 shows that using MATLAB software carries out simulation for the virtual sound signals, then through the neural network training on signal, finally we can get the multi modal response curve of virtual language. It can be seen from the chart that the multi modal response of language has mainly three main peaks, and through the simulation it also proves that language multi modal has higher response for sound, image and text. In the relevance analysis of language mode, we need to consider the language of visual, auditory and tactile mode improving the ability of language application.

\section{Summary}

(1) This paper designs the neural network algorithm of virtual language multimodal training and the voice analysis toolbox of using MATLAB software carries on programming for the modal algorithm of language, which can obtain three kinds of virtual language mode signal.

(2) We carry on processing for the three mode signal of virtual language, and then using numerical laboratory carry on processing simulation calculation for the modal language, at the same time 
combined with language sound, image and text analysis, we can get the response contours and curves of virtual language auditory, tactile and visual.

(3) Although the verification of neural network analysis algorithm functions in the virtual language multi modal analysis, we have not compared with other algorithms. So, we should be combined with a variety of algorithms in the future research, to find the most suitable language modal analysis algorithm.

\section{Acknowledgments}

The work was supported by the following two projects. One is from the joint fund project of Philosophy Social Sciences and Foreign of Hunan Province in 2013 with the project number13WLH39 of [2013]29 (Hunan Academy of social sciences ) and the project name Study on Dynamic Multimodal Discourse of English Based on ELAN. The other is from the teaching reform project of Hunan Province Education Department in 2012 with the project number 2012-401-465 and the project name Teaching Model of Reading and Writing for College English Based on Systemic Functional Linguistics.

\section{References}

[1] D.Z. Feng. Digital technology and multimodal discourse analysis. Journal of Beijing Science and Technology University, 2011(4): 112-113.

[2] D.L. Zhang. Design of multimodal foreign language teaching and modal call. China language, 2011(3): 45-46.

[3] Q. Liu, M.W. Pan. Research on the construction of science students' English spoken multimodal corpus. Modern educational technology, 2011(4): 67-68.

[4] D.L. Zhang. Application of multimodal discourse theory and media technology in foreign language teaching. Foreign language teaching, 2011(4): 89-92.

[5] Q. Liu, M.W. Pan. Study on college students' English oral non verbal communicative competence under the multimodal environment. Foreign language teaching, 2011(2): 56-57.

[6] X.Z. Yang. Multimodal discourse analysis and systemic functional linguistics. Foreign language teaching, 2012(4): 78-80.

[7] D.L. Zhang. Application of multimodal discourse theory and media technology in foreign language teaching. Foreign language teaching, 2012(4): 25-27.

[8] Q.H. Wei. Research on multi modal and college students' multiliteracies. Foreign language teaching research, 2011(2): 34-36.

[9] R. Gao, B.L. Zhao. Study of college English classroom teaching discourse. Border economy and culture, 2011(12): 102-104.

[10] D.L. Zhang, S.J. Zhang. Explore of multimodal foreign language textbooks compiling principle. Foreign language field, 2011(5): 56-58.

[11] C.Y. Yuan. Exploration on multi modal information cognition teaching and learning mode-the complex course "legal English" teaching reform. Shandong foreign language teaching, 2011(4): 120-123. 